\title{
Management Strategies for Elderly Patients with Diffuse Large B-Cell Lymphoma
}

\author{
Loretta J Nastoupil, Rajni Sinha, and Christopher R. Flowers \\ Department of Hematology and Oncology, Winship Cancer Institute, 1365 Clifton Road, N.E. \\ Building B, Suite 4302, Emory University, Atlanta, GA 30322
}

\section{INTRODUCTION}

Diffuse large B-cell lymphoma (DLBCL) is the most commonly occurring form of NonHodgkin lymphoma (NHL), accounting for approximately one-third of adult lymphomas. There was an unprecedented rise in NHL incidence (3-4\% per year) from the 1970s to the mid-1990s, comparable only to the rise in skin cancers ${ }^{1}$. It is estimated that there will be 70,140 new cases in 2012, making NHL the $7^{\text {th }}$ most common cancer ${ }^{2}$. Though there appears to be a stabilization in the incidence of lymphoma since the late 1990s for young patients, this does not appear to be true for elderly patients, the majority of whom present over the age of $65^{3,4}$. The median age of the world's population is increasing, with the proportion of the United States (US) population aged $\geq 65$ years projected to increase from $12.4 \%$ in 2000 to $19.6 \%$ in $2030^{5}$. It is expected that the prevalence of NHL will increase with the aging population and it is well recognized that this patient population would benefit from the most appropriate modern therapy.

Though heterogeneous, DLBCL has an aggressive natural history with a median survival of one year in untreated patients ${ }^{6}$. In the majority of studies conducted before 2000 , older patients were systematically considered to be poor candidates for standard therapy and were treated with low-dose regimens ${ }^{7,8}$. The dilemma in managing elderly patients is that with aggressive treatment DLBCL is curable for the majority of patients, however, providing adequate therapy to this patient population can be complicated by co-morbid conditions, decreased drug metabolism, and worse performance status when compared to younger patients. Herein, we examine emerging trends on the clinical management and biology of DLBCL in the elderly patient population and explore findings from recent meetings and publications that will aid in further defining our management of this disease.

\section{AGE, CLINICAL OR BIOLOGIC PREDICTOR}

Age has been one of the most important adverse prognostic features in NHL. Numerous studies have associated older age with inferior outcomes ${ }^{9-12}$. Age, defined as $>60$ years, is a component of the international prognostic index (IPI), developed in the 1990s, as a clinical tool to predict outcome for patients with DLBCL ${ }^{13}$. In addition to age, clinical features that were predictive of overall survival (OS) and relapse-free survival (RFS) were derived from a pooled analysis of more than 2000 patients with aggressive lymphoma (mainly DLBCL) treated with an anthracycline-containing chemotherapy regimen between 1982-1987. These factors include: advanced stage, elevated serum lactate dehydrogenase (LDH), poorer performance status, and number of extranodal disease sites $\geq 2$. Patients are scored based on the presence of these clinical features and can then be risk stratified into one of four discrete prognostic groups with 5-year predicted survival rates of $73 \%, 51 \%, 43 \%$, and $26 \%$. A more recent analysis of more than 1000 patients treated with chemoimmunotherapy suggests that IPI maintains its relevance in the rituximab era and remains a valid prognostic tool ${ }^{14}$. An alternative index, the Elderly IPI (E-IPI) with an age cut off of 70 years rather than 60 has 
been reported to provide better discrimination in outcome for patients $>60$ years with DLBCL treated with R-CHOP ${ }^{15}$. These findings were recently validated when the conventional IPI and the E-IPI were compared in the RICOVER-60 data set. Both the IPI and E-IPI provided prognostic discrimination for OS; however, the E-IPI outperformed the conventional IPI ${ }^{16}$.

Although age is a relevant clinical predictor of outcome, is it a reflection of a patient characteristic or an intrinsic biological feature of the tumor? Over the past decade there have been significant efforts to define biologically relevant subgroups of DLBCL. Geneexpression profiling (GEP) from DNA microarrays has delineated expression patterns that reflect differences in cell of origin, proliferation rate, and host immune response to the tumor $^{17}$. DLBCL can now be subdivided using GEP into at least three molecular subtypes: activated B-cell-like (ABC), germinal center B-cell-like (GCB), and primary mediastinal large B-cell lymphoma (PMBL). The GCB and $\mathrm{ABC}$ signatures are biologic determinants of prognosis in patients treated with chemoimmunotherapy, independent of IPI, with the GCB subtype having a significantly better five-year survival rate than ABC DLBCL (OS 60\% vs. $35 \%, \mathrm{P}<0.001)^{18}$.

Whether there is a relationship between aging and the distribution of these two gene expression profiles has not been clearly defined. Rosenwald et al. reported a trend towards a higher proportion of $\mathrm{ABC}$ subtype in patients over the age of 60 years ${ }^{19}$. A retrospective analysis studied this further. GEP was performed on 131 de novo DLBCL patients $>50$ years of age (median 68 years, range 50-91) and reported a significant increase in ABC subtype in proportion to increasing age ${ }^{20}$. To strengthen their results, a similar analysis was done on data accessed from the Lenz study ${ }^{21}$. Again, they found a similar increase in the proportion of $\mathrm{ABC}$ subtype associated with age (average increase of $13.7 \%$ in $\mathrm{ABC}$ for every 10 years after age 50 vs. $7.5 \%$ in the Lenz series) ${ }^{20}$. In contrast to adults, children with DLBCL have excellent prognosis. Oschlies et al. found a striking predominance of GCB in 63 cases of pediatric DLBCL which may explain their better outcomes and again suggests a relationship between DLBCL subtype distribution and age ${ }^{22}$.

To counter these claims, a retrospective analysis of very elderly patients (> 80 years) reported that clinical and biologic characteristics of older patients with lymphoma at presentation are similar to younger patients, but there were significant differences in disease management ${ }^{8}$. No specific chromosomal or genetic abnormalities have been described in elderly patients; however, the majority of studies have been focused on defining the level of heterogeneity between different subtypes not heterogeneity between age groups. Further speculation on the pathogenesis of poor prognosis in elderly patients with DLBCL includes the finding that B-cell diversity decreases dramatically with age, and this loss of diversity is characterized by clonal expansion of B-cells in vivo ${ }^{23}$. A well-recognized predisposing risk factor for the occurrence of NHL is immunosuppression ${ }^{24}$. Host factors that have been implicated in this increased risk include a high prevalence of EBV and defects in immune regulation with resultant cytokine production. EBV-related DLBCL are almost exclusively reported in the elderly, including very old patients and portends a poor prognosis with a median survival of two years ${ }^{25}$. Further study into the relationship of age and tumor biology is warranted.

\section{ASSESSING THERAPEUTIC OPTIONS FOR ELDERLY PATIENTS}

One of the challenges faced with this particular patient population are age-related factors that must be considered when discussing treatment options with patients. It is well known that with increasing age, the likelihood of co-morbid disease also increases. For example, in one survey, $61 \%$ of the patients 70 years or older had at least one co-morbid condition 
compared with $20 \%$ of patients younger than 60 years ${ }^{26}$. Of particular importance is the prevalence of heart disease which may hinder therapeutic options such as anthracyclines. Co-morbid conditions also frequently results in polypharmacy. Elderly patients may have alterations in drug absorption, distribution, metabolism, and clearance which alters the pharmacodynamics of therapeutic agents and attention to appropriate dosing of drugs is imperative ${ }^{27}$. Hematopoietic reserve is also different from younger patients and myelotoxicity can be increased with standard regimens ${ }^{28}$.

Determining performance status, though subjective, carries prognostic implications, assessing the degree of functional dependence and frailty in elderly patients is important. Age and performance status are currently the most frequently used criteria to select patients for standard chemotherapy, but the final decision is generally left to the clinical judgment of the treating physician ${ }^{29}$. There is a large body of geriatric literature that tries to address this problem of subjectivity by designing specific comprehensive geriatric assessment scores as a more effective and objective tool than clinical decision making. Though, the use of such assessments has not been shown to affect OS. Assessing the tolerability of systemic therapy in elderly patients is complex. Clinicians must be objective about the process as decreasing dosage or withholding potentially curative therapy for putative increased toxicity results in worse outcomes ${ }^{30,31}$.

\section{DLBCL TREATMENT AND OUTCOMES IN THE ELDERLY}

Developed in the 1970's, the anthracycline-based chemotherapy regimen

cyclophosphamide, doxorubicin, vincristine, and prednisone (CHOP) was found to be better tolerated and have similar OS to more intensive regimens leading CHOP to become the standard therapy for DLBCL ${ }^{32,33}$. Several attempts to decrease the chemotherapy doses in the standard CHOP regimen or substitute less toxic drugs in the combination for elderly patients have decreased toxicity but did not improve survival ${ }^{11,34,35}$. An important development in the treatment of aggressive lymphomas occurred when rituximab (R) demonstrated activity in phase II studies, ${ }^{36,37}$ leading to the development of phase III trials across the world comparing R-CHOP to standard CHOP. One of the earliest reports of survival benefit from the addition of $\mathrm{R}$ was from the Groupe d'Etude des Lymphomas de l'Adulte (GELA) demonstrating that patients over the age of 60 years with DLBCL treated with R-CHOP had superior outcomes compared to patients treated with CHOP where both regimens were given every 21 days ${ }^{38}$. This study did not include patients over 80 years of age but did show a similar benefit for patients aged 60-70 years, 71-75 years, and 76-80 years ${ }^{39}$. A US Intergroup trial in older patients (> 60 years), randomized to R-CHOP-21 or CHOP-21 with a second randomization in responders to maintenance $\mathrm{R}$ or observation demonstrated similar results ${ }^{40,41}$. The 3-year failure-free survival rate was $53 \%$ for R-CHOP patients and $46 \%$ for CHOP patients $(\mathrm{P}=0.04)$ and no benefit was seen with maintenance $\mathrm{R}$ in the R-CHOP arm. R-CHOP has been well established as the standard induction regimen in DLBCL.

Given improvement in outcomes with the addition of R, the German High Grade NonHodgkin Lymphoma Study Group (DSHNHL) explored a dose-intense regimen incorporating $\mathrm{R}$ based on previous data demonstrating improved outcomes in older patients treated with CHOP given every 14 days $(\mathrm{CHOP}-14)^{42}$. The RICOVER-60 trial randomized elderly (61-80 years) patients to receive 6 or 8 cycles of CHOP-14 with or without R. Six cycles of R-CHOP-14 significantly improved EFS, PFS, and OS in comparison to 6 cycles of CHOP-14. In addition, 6 cycles of R-CHOP-14 was superior to 8 cycles of RCHOP- $14{ }^{43}$. This established R-CHOP-14 as the preferred regimen for elderly patients in Germany. A phase II study also assessed the feasibility and efficacy of a biweekly regimen, R-COMP-14 (rituximab, cyclophosphamide, non-pegylated liposome-encapsulated 
doxorubicin, vincristine and prednisone) in elderly patients (median age 73, range 62-82) with poor-risk DLBCL who also had moderate to high cardiac comorbidity ${ }^{44}$. Times to progression and OS at 4-years were $77 \%$ and $67 \%$, respectively. The Age-adjusted Charlson Comorbidity Index (aaCCI) correlated with treatment failures with patients scoring $\leq 7$ having a longer time to treatment failure ( $66 \%$ vs. $29 \% ; \mathrm{P}=0.009)$. Incidence of cardiac grade $3-5$ adverse events was $17 \%$. This demonstrates that R-COMP-14 is feasible in patients with cardiac co-morbidity.

The results of a randomized study comparing R-CHOP-14 to the standard R-CHOP-21 were presented by at the American Society of Clinical Oncology (ASCO) conference in $2011^{45}$. In this trial, DLBCL patients (52\% > 60 years of age) were randomized to either 8 cycles of standard R-CHOP-21 or 6 cycles of R-CHOP-14 plus granulocyte colony-stimulating factor (G-CSF) with two additional cycles of single agent R (similar to RICOVER-60). Overall response rates were similar between the 2 arms, and after a median follow-up of 37 months, PFS and OS were not significantly different. Subgroup analyses failed to identify a population of patients that benefited from the dose-dense R-CHOP. In particular, there was no benefit for R-CHOP-14 in the older age subgroup of DLBCL patients. At the 2011 American Society of Hematology (ASH) meeting, Dr. Pfreundschuh described a pharmacokinetically-optimized schedule of R-CHOP-14 that achieves high R levels early and maintains optimal serum levels over a prolonged period of time with 8 doses of $R$ (SMARTE-R-CHOP-14) ${ }^{46}$. This trial showed a benefit of SMARTE-R-CHOP-14 when compared to historical controls (RICOVER-60) and suggested that prior trials of RCHOP-14 may have been compromised by the shorter exposure to $\mathrm{R}$ when 8 applications are given every 2 weeks. However, randomized data are needed to determine whether pharmacokinetically-optimized R-CHOP-14 may provide benefits for elderly patients over R-CHOP-21.

Important to highlight is the reference to elderly patients in these trials, often defined by older than 60 years of age and less than 80 years. GELA has performed a prospective, multicenter, phase II study of low-dose CHOP chemotherapy regimen and R in patients aged 80 years and older (median 83 , range $80-95$ ) with DLBLC ${ }^{47}$. Patients received 6 cycles of R-miniCHOP $\left(375 \mathrm{mg} / \mathrm{m}^{2}\right.$ rituximab, $400 \mathrm{mg} / \mathrm{m}^{2}$ cyclophosphamide, $25 \mathrm{mg} / \mathrm{m}^{2}$ doxorubicin, and $1 \mathrm{mg}$ vincristine on day 1 of each cycle, and $40 \mathrm{mg} / \mathrm{m}^{2}$ prednisone on days $1-5)$ at 3 week intervals. The intention to treat population included 149 patients. After a median follow-up of 20 months, median OS was 29 months, 2-year OS was 59\%. Median progression-free survival (PFS) was 21 months, with a 2-year PFS of $47 \%$. The majority of deaths $(57 \%)$ were secondary to lymphoma progression, 12 of $58(21 \%)$ deaths were attributed to treatment toxicity. The most frequent side-effect was hematological toxicity (grade $\geq 3$ neutropenia 40\%, febrile neutropenia 7\%). Prophylactic G-CSF was left to the discretion of the treating physician. However, in the event of severe neutropenia or neutropenic fever, G-CSF was recommended from day 6 to 13 of the subsequent cycle or until neutrophils were $\geq 1.0 \times 10^{9} / \mathrm{L}$. In multivariate analysis, the only parameter associated with worse outcome was low serum albumin concentration. Included in the initial assessment was the instrumental activities of daily living (IADL) scale. Without systematic comparison and the absence of a control arm, this study suggests that in selected patients older than 80 years with a good performance status (0-2), R-miniCHOP offers a compromise between efficacy and safety. However, PFS and OS at 2-years in this trial was lower than would be expected for standard CHOP-21 in patients $>60$ years, thus, this approach would benefit from comparison to standard CHOP-21 in this population given that most deaths were due to lymphoma progression. 


\section{FUTURE DIRECTIONS}

Prospective studies directed at elderly patients are needed particularly for guiding treatment decisions. A particular area of need is in the relapsed or refractory setting. Younger patients who experience relapse will be evaluated for salvage therapy and high-dose therapy (HDT) with autologous stem cell transplant (ASCT). With better supportive care, consideration of HDT-ASCT in those who are medically fit is an option ${ }^{48-50}$. Standard salvage therapy can be difficult to administer in this patient population due to the feasibility of treatment with platinum therapy with diminished creatinine clearance. Given the limited options for elderly patients, referral for clinical trials is appropriate for many patients. Several small molecule inhibitors, antibodies, and other approaches are under study, and these therapies may provide the advantage of minimal toxicity ${ }^{51}$.

Several novel agents are under investigation many of which are rationally designed from knowledge gained from GEP analysis. Enzastaurin, a protein kinase c-beta inhibitor has been demonstrated in phase II study to prolong PFS in a subset of patients ${ }^{52}$ and a phase III trial incorporating this drug in first remission maintenance has been completed and is awaiting analysis. Several other pathway inhibitors have demonstrated activity in DLBCL targets include spleen tyrosine kinase (syk), Brutons tyrosine kinase, PI3K, and mTOR. Navitoclax is a high affinity small molecule inhibitor of the anti-apoptotic activity of BCL-2 and BCL-XL under investigation in DLBCL. Studies of aurora kinase inhibitors both as single agents and in combination are ongoing, based on the finding that aurora kinases are upregulated by $\mathrm{c}-\mathrm{Myc}^{53}$. Overexpression of NF- $\mathrm{\kappa B}$ is a potential target of the ABC subtype of DLBCL and bortezomib has been used to exploit this concept ${ }^{54}$. Lenalidomide has shown significant activity in relapsed DLBCL, again higher responses are seen in the non-GCB subtype $^{55}$. As discussed these strategies hold promise for elderly patients as most appear to have limited toxicity or toxicity that does not overlap considerably with prior chemotherapy.

\section{CONCLUSIONS}

Though a number of retrospective analyses have been performed reporting outcomes in elderly patients, prospective data is limited. With the aging population, the prevalence of DLCBL particularly in elderly patients is expected to increase. Future study is needed not only to address treatment strategies but to define the biologic heterogeneity between younger and older patients with DLBCL so that more rational therapeutic design can be investigated. Several issues arise when addressing worse outcomes seen with elderly patients compared to their younger counterparts. Elderly patients often have more co-morbid illnesses, worse performance status, less hematologic reserve, and altered pharmacokinetics related to decreased metabolism and clearance of drugs. Clinical judgment, clearly contributes to determination as to whether patients are fit for aggressive treatment. Since it is well established that aggressive chemoimmunotherapy can provide cure to elderly patients, what is needed are objective tools to identify patients who safely benefit from aggressive therapy. Currently, there are no validated methods to prospectively identify elderly patients fit enough to receive the same treatment as younger patients. The first question that should be asked by providers is whether or not their patient can be treated with R-CHOP as this is the current standard induction therapy for medically fit patients. For those who are not candidates for R-CHOP, prospective data support the use of R-miniCHOP or R-COMP-14 as possible alternatives. Supportive measures such as prophylaxis with G-CSF are recommended. Novel agents that are currently under investigation are promising in that they offer a rational approach for biologically-targeted therapy with the advantage of improved tolerability. Prospective studies are needed that include elderly patients (including those over the age of 80) to further guide treatment decisions. 


\section{Acknowledgments}

This work was supported by the Georgia Cancer Coalition Distinguished Scientist Award and Cancer Research Award, by an American Society of Hematology Amos Medical Faculty Development Award, by National Cancer Institute R21 CA158686-01A1, and by funding from a Leukemia and Lymphoma Society Translational Research Award.

\section{References}

1. Flowers CR, Sinha R, Vose JM. Improving outcomes for patients with diffuse large B-cell lymphoma. CA Cancer J Clin. 2010; 60:393-408. [PubMed: 21030533]

2. Siegel R, Naishadham D, Jemal A. Cancer statistics, 2012. CA Cancer J Clin. 2012; 62:10-29. [PubMed: 22237781]

3. Hartge P, Devesa SS. Quantification of the impact of known risk factors on time trends in nonHodgkin's lymphoma incidence. Cancer Res. 1992; 52:5566s-5569s. [PubMed: 1394175]

4. Howe HL, Wingo PA, Thun MJ, et al. Annual report to the nation on the status of cancer (1973 through 1998), featuring cancers with recent increasing trends. J Natl Cancer Inst. 2001; 93:824-42. [PubMed: 11390532]

5. Bureau. USC. International database. Table 094. Midyear population, by age and sex. Available at http://www.census.gov/population/www/projections/natdet-D1A.html

6. Fisher RI, Miller TP, O’Connor OA. Diffuse aggressive lymphoma. Hematology (Am Soc Hematol Educ Program). 2004:221-36. [PubMed: 15561685]

7. Coiffier B. What treatment for elderly patients with aggressive lymphoma? Ann Oncol. 1994; 5:873-5. [PubMed: 7696157]

8. Thieblemont C, Grossoeuvre A, Houot R, et al. Non-Hodgkin's lymphoma in very elderly patients over 80 years. A descriptive analysis of clinical presentation and outcome. Ann Oncol. 2008; 19:774-9. [PubMed: 18065404]

9. Dixon DO, Neilan B, Jones SE, et al. Effect of age on therapeutic outcome in advanced diffuse histiocytic lymphoma: the Southwest Oncology Group experience. J Clin Oncol. 1986; 4:295-305. [PubMed: 3512783]

10. Vose JM, Armitage JO, Weisenburger DD, et al. The importance of age in survival of patients treated with chemotherapy for aggressive non-Hodgkin's lymphoma. J Clin Oncol. 1988; 6:183844. [PubMed: 2462026]

11. Bastion Y, Blay JY, Divine M, et al. Elderly patients with aggressive non-Hodgkin's lymphoma: disease presentation, response to treatment, and survival--a Groupe d'Etude des Lymphomes de l'Adulte study on 453 patients older than 69 years. J Clin Oncol. 1997; 15:2945-53. [PubMed: 9256139]

12. Gomez H, Mas L, Casanova L, et al. Elderly patients with aggressive non-Hodgkin's lymphoma treated with CHOP chemotherapy plus granulocyte-macrophage colony-stimulating factor: identification of two age subgroups with differing hematologic toxicity. J Clin Oncol. 1998; 16:2352-8. [PubMed: 9667250]

13. A predictive model for aggressive non-Hodgkin's lymphoma. The International Non-Hodgkin's Lymphoma Prognostic Factors Project. N Engl J Med. 1993; 329:987-94. [PubMed: 8141877]

14. Ziepert M, Hasenclever D, Kuhnt E, et al. Standard International prognostic index remains a valid predictor of outcome for patients with aggressive CD20+ B-cell lymphoma in the rituximab era. $\mathrm{J}$ Clin Oncol. 2010; 28:2373-80. [PubMed: 20385988]

15. Advani RH, Chen H, Habermann TM, et al. Comparison of conventional prognostic indices in patients older than 60 years with diffuse large B-cell lymphoma treated with R-CHOP in the US Intergroup Study (ECOG 4494, CALGB 9793): consideration of age greater than 70 years in an elderly prognostic index(E-IPI). Br J Haematol. 2010; 151:143-51. [PubMed: 20735398]

16. Advani RH, Hong F, et al. Validation of the Elderly IPI (E-IPI) for Patients with Diffuse Large B Cell Lymphoma Using An Independent Data Set From the RICOVER-60 Trial of the German High-Grade Non-Hodgkin Lymphoma Study Group (DSHNHL). ASH Annual Meeting Abstracts. 2011; 118(21):1568. 
17. Alizadeh AA, Eisen MB, Davis RE, et al. Distinct types of diffuse large B-cell lymphoma identified by gene expression profiling. Nature. 2000; 403:503-11. [PubMed: 10676951]

18. Rimsza LM, LeBlanc ML, Unger JM, et al. Gene expression predicts overall survival in paraffinembedded tissues of diffuse large B-cell lymphoma treated with R-CHOP. Blood. 2008; 112:3425-3433. [PubMed: 18544678]

19. Rosenwald A, Wright G, Chan WC, et al. The use of molecular profiling to predict survival after chemotherapy for diffuse large-B-cell lymphoma. N Engl J Med. 2002; 346:1937-47. [PubMed: 12075054]

20. Mareschal S, Lanic H, Ruminy P, et al. The proportion of activated B-cell like subtype among de novo diffuse large B-cell lymphoma increases with age. Haematologica. 2011; 96:1888-90. [PubMed: 21859735]

21. Lenz G, Wright G, Dave SS, et al. Stromal gene signatures in large-B-cell lymphomas. N Engl J Med. 2008; 359:2313-23. [PubMed: 19038878]

22. Oschlies I, Klapper W, Zimmermann M, et al. Diffuse large B-cell lymphoma in pediatric patients belongs predominantly to the germinal-center type B-cell lymphomas: a clinicopathologic analysis of cases included in the German BFM (Berlin-Frankfurt-Munster) Multicenter Trial. Blood. 2006; 107:4047-52. [PubMed: 16424389]

23. Gibson KL, Wu YC, Barnett Y, et al. B-cell diversity decreases in old age and is correlated with poor health status. Aging Cell. 2009; 8:18-25. [PubMed: 18986373]

24. Fisher SG, Fisher RI. The epidemiology of non-Hodgkin's lymphoma. Oncogene. 2004; 23:652434. [PubMed: 15322522]

25. Wong HH, Wang J. Epstein-Barr virus positive diffuse large B-cell lymphoma of the elderly. Leuk Lymphoma. 2009; 50:335-40. [PubMed: 19255922]

26. van Spronsen DJ, Janssen-Heijnen ML, Breed WP, et al. Prevalence of co-morbidity and its relationship to treatment among unselected patients with Hodgkin's disease and non-Hodgkin's lymphoma,1993-1996. Ann Hematol. 1999; 78:315-9. [PubMed: 10466443]

27. Lichtman SM. Chemotherapy in the elderly. Semin Oncol. 2004; 31:160-74. [PubMed: 15112147]

28. Balducci L, Repetto L. Increased risk of myelotoxicity in elderly patients with non-Hodgkin lymphoma. Cancer. 2004; 100:6-11. [PubMed: 14692018]

29. Tucci A, Ferrari S, Bottelli C, et al. A comprehensive geriatric assessment is more effective than clinical judgment to identify elderly diffuse large cell lymphoma patients who benefit from aggressive therapy. Cancer. 2009; 115:4547-53. [PubMed: 19562776]

30. Coiffier B. Treatment paradigms in aggressive non-Hodgkin's lymphoma in elderly patients. Clin Lymphoma. 2002; 3 (Suppl 1):S12-8. [PubMed: 12521384]

31. Lyman GH, Dale DC, Friedberg J, et al. Incidence and predictors of low chemotherapy doseintensity in aggressive non-Hodgkin's lymphoma: a nationwide study. J Clin Oncol. 2004; 22:4302-11. [PubMed: 15381684]

32. Fisher RI, Gaynor ER, Dahlberg S, et al. Comparison of a standard regimen (CHOP) with three intensive chemotherapy regimens for advanced non-Hodgkin's lymphoma. N Engl J Med. 1993; 328:1002-6. [PubMed: 7680764]

33. Flowers CR, Armitage JO. A decade of progress in lymphoma: advances and continuing challenges. Clin Lymphoma Myeloma Leuk. 2010; 10:414-23. [PubMed: 21156459]

34. Bessell EM, Burton A, Haynes AP, et al. A randomised multicentre trial of modified CHOP versus MCOP in patients aged 65 years andover with aggressive non-Hodgkin's lymphoma. Ann Oncol. 2003; 14:258-67. [PubMed: 12562653]

35. Osby E, Hagberg H, Kvaloy S, et al. CHOP is superior to CNOP in elderly patients with aggressive lymphoma while outcome is unaffected by filgrastim treatment: results of a Nordic Lymphoma Group randomized trial. Blood. 2003; 101:3840-8. [PubMed: 12531794]

36. Coiffier B, Haioun C, Ketterer N, et al. Rituximab (anti-CD20 monoclonal antibody) for the treatment of patients with relapsing or refractory aggressive lymphoma: A multicenter phase II study. Blood. 1998; 92:1927-1932. [PubMed: 9731049]

37. Vose JM, Link BK, Grossbard ML, et al. Phase II study of rituximab in combination with chop chemotherapy in patients with previously untreated, aggressive non-Hodgkin's lymphoma. J Clin Oncol. 2001; 19:389-97. [PubMed: 11208830] 
38. Coiffier B, Lepage E, Briere J, et al. CHOP chemotherapy plus rituximab compared with CHOP alone in elderly patients with diffuse large-B-cell lymphoma. N Engl J Med. 2002; 346:235-42. [PubMed: 11807147]

39. Feugier P, Van Hoof A, Sebban C, et al. Long-term results of the R-CHOP study in the treatment of elderly patients with diffuse large B-cell lymphoma: a study by the Groupe d'Etude des Lymphomes de l'Adulte. J Clin Oncol. 2005; 23:4117-26. [PubMed: 15867204]

40. Habermann TM, Weller EA, Morrison VA, et al. Rituximab-CHOP versus CHOP alone or with maintenance rituximab in older patients with diffuse large B-cell lymphoma. J Clin Oncol. 2006; 24:3121-7. [PubMed: 16754935]

41. Mihelic R, Kaufman J, Lonial S, et al. Maintenance therapy in lymphoma. Clin Lymphoma Myeloma. 2007; 7:507-13. [PubMed: 18021467]

42. Pfreundschuh M, Trumper L, Kloess M, et al. Two-weekly or 3-weekly CHOP chemotherapy with or without etoposide for the treatment of elderly patients with aggressive lymphomas: results of the NHL-B2 trial of the DSHNHL. Blood. 2004; 104:634-41. [PubMed: 15016643]

43. Pfreundschuh M, Schubert J, Ziepert M, et al. Six versus eight cycles of bi-weekly CHOP-14 with or without rituximab in elderly patients with aggressive CD20+ B-cell lymphomas: a randomised controlled trial (RICOVER-60). Lancet Oncology. 2008; 9:105-116. [PubMed: 18226581]

44. Corazzelli G, Frigeri F, Arcamone M, et al. Biweekly rituximab, cyclophosphamide, vincristine, non-pegylated liposome-encapsulated doxorubicin and prednisone (R-COMP-14) in elderly patients with poor-risk diffuse large B-cell lymphoma and moderate to high 'life threat' impact cardiopathy. Br J Haematol. 2011; 154:579-89. [PubMed: 21707585]

45. Cunningham DSP, Mouncey P, Qian W, Jack AS, Pocock C, et al. R-CHOP14 versus R-CHOP21: Result of a randomized phase III trial for the treatment of patients with newly diagnosed diffuse large B-cell non-Hodgkin lymphoma. Journal of Clinical Oncology. 2011; 29:Abstract \#8000.

46. Pfreundschuh MHG, Zeynalova S, et al. Improved Outcome of Elderly Poor-Prognosis DLBCL Patients with 6xCHOP-14 and 8 Applications of Rituximab (R) Given Over An Extended Period: Results of the SMARTE-R-CHOP-14 Trial of the German High-Grade Non-Hodgkin Lymphoma Study Group (DSHNHL). ASH Annual Meeting Abstracts. 2011; 118:592.

47. Peyrade F, Jardin F, Thieblemont C, et al. Attenuated immunochemotherapy regimen (RminiCHOP) in elderly patients older than 80 years with diffuse large B-cell lymphoma: a multicentre, single-arm, phase 2 trial. Lancet Oncol. 2011; 12:460-8. [PubMed: 21482186]

48. Gopal AK, Gooley TA, Golden JB, et al. Efficacy of high-dose therapy and autologous hematopoietic stem cell transplantation for non-Hodgkin's lymphoma in adults 60 years of age and older. Bone Marrow Transplant. 2001; 27:593-9. [PubMed: 11319588]

49. Jantunen E, Itala M, Siitonen T, et al. Autologous stem cell transplantation in patients with chronic lymphocytic leukaemia: the Finnish experience. Bone Marrow Transplant. 2006; 37:1093-8. [PubMed: 16699533]

50. Zallio F, Cuttica A, Caracciolo D, et al. Feasibility of peripheral blood progenitor cell mobilization and harvest to support chemotherapy intensification in elderly patients with poor prognosis: nonHodgkin's lymphoma. Ann Hematol. 2002; 81:448-53. [PubMed: 12224002]

51. Sinha R, DeJoubner N, Flowers C. Novel agents for diffuse large B-cell lymphoma. Expert Opin Investig Drugs. 2011; 20:669-80.

52. Robertson MJ, Kahl BS, Vose JM, et al. Phase II study of enzastaurin, a protein kinase C beta inhibitor, in patients with relapsed or refractory diffuse large B-cell lymphoma. J Clin Oncol. 2007; 25:1741-6. [PubMed: 17389337]

53. denHollander J, Rimpi S, Doherty JR, et al. Aurora kinases A and B are up-regulated by Myc and are essential for maintenance of the malignant state. Blood. 2010; 116:1498-505. [PubMed: 20519624]

54. Ruan J, Martin P, Furman RR, et al. Bortezomib plus CHOP-rituximab for previously untreated diffuse large B-cell lymphoma and mantle cell lymphoma. J Clin Oncol. 2011; 29:690-7. [PubMed: 21189393]

55. Hernandez-Ilizaliturri FJ, Deeb G, Zinzani PL, et al. Higher response to lenalidomide in relapsed/ refractory diffuse large B-cell lymphoma in nongerminal center B-cell-like than in germinal center B-cell-like phenotype. Cancer. 2011; 117:5058-66. [PubMed: 21495023] 